\title{
Papers
}

\section{Fluid resuscitation with colloid or crystalloid solutions in critically ill patients: a systematic review of randomised trials}

Gill Schierhout, Ian Roberts

\begin{abstract}
Objective: To determine the effect on mortality of resuscitation with colloid solutions compared with resuscitation with crystalloids.

Design: Systematic review of randomised controlled trials of resuscitation with colloids compared with crystalloids for volume replacement of critically ill patients; analysis stratified according to patient type and quality of allocation concealment.

Subjects: 37 randomised controlled trials were eligible, of which 26 unconfounded trials compared colloids with crystalloids $(n=1622)$. (The 10 trials that compared colloid in hypertonic crystalloid with isotonic crystalloid $(n=1422)$ and one trial that compared colloid in isotonic crystalloid with hypertonic crystalloid $(\mathrm{n}=38)$ are described in the longer version on our website www.bmj.com).

Main outcome measures: Mortality from all causes at end of follow up for each trial.

Results: Resuscitation with colloids was associated with an increased absolute risk of mortality of $4 \%$ (95\% confidence interval $0 \%$ to $8 \%$ ), or four extra deaths for every 100 patients resuscitated. The summary effect measure shifted towards increased mortality with colloids when only trials with adequate concealment of allocation were included. There was no evidence for differences in effect among patients with different types of injury that required fluid resuscitation.
\end{abstract}

Conclusions: This systematic review does not support the continued use of colloids for volume replacement in critically ill patients.

\section{Introduction}

Fluid resuscitation for hypovolaemia is integral to the acute medical management of critically ill patients. Although recent studies have suggested that the timing of volume replacement deserves careful consideration, ${ }^{1}$ when it comes to selecting the resuscitation fluid doctors are faced with a range of options. At the most basic level the choice is between a colloid or crystalloid solution. Colloids are widely used, having been recommended in a number of resuscitation guidelines and intensive care management algorithms. ${ }^{23}$ The American hospital consortium guidelines recommend that colloids are used in haemorrhagic shock until blood products become available and in non-haemorrhagic shock after an initial infusion with crystalloid. A 1995 survey of American academic health centres, however, found that the use of colloids far exceeded these recommendations. ${ }^{4}$ Surveys of burn care in the United States $^{5}$ and Australia ${ }^{6}$ found that the use of colloids for resuscitation varied without a set pattern. The choice of fluid has considerable cost implications: volume replacement with colloids is considerably more expensive than with crystalloids.

Clinical studies have shown that colloids and crystalloids have different effects on a range of important physiological parameters. Because of these differences, mortality from all causes is arguably the most clinically relevant outcome measure in randomised trials comparing the two fluid types. Although there have been meta-analyses of mortality in randomised trials comparing colloids and crystalloids, ${ }^{78}$ neither of these satisfies the criteria that have been proposed for systematic reviews ${ }^{9}$ and they predate most of the trials that have been conducted with synthetic colloids and hypertonic crystalloid solutions. The purpose of this review was to identify and synthesise all available unconfounded evidence of the effect on mortality in critically ill patients of colloids compared with crystalloids for volume replacement.

\section{Methods}

\section{Identification of trials}

Our aim was to identify all relevant randomised controlled trials available for review by June 1997. Relevant trials were those in which critically ill patients (excluding neonates) who required fluid resuscitation were assigned to colloid or crystalloid resuscitation protocols on the basis of random or quasi-random allocation. If the allocation procedure could not be fully ascertained from the published report, the author was contacted for clarification.

We included trials in which participants were critically ill as a result of trauma or burns, were undergoing surgery, or had other critical conditions such as complications of sepsis. Trials were considered unconfounded if one treatment group differed from another only in the treatment of interest. Thus a trial that compared colloid and hypertonic crystalloid with hyper-

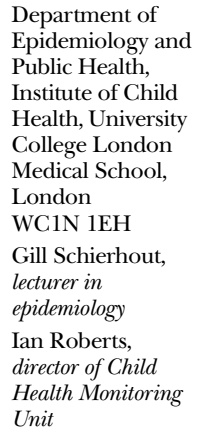

Unit

Correspondence to: Dr Schierhout

BMJ 1998;316:961-4

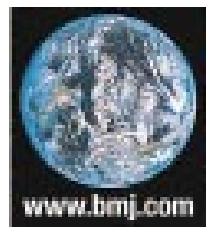

A longer version of this article is available on our website 
tonic crystalloid would be included, as would a trial which compared colloid and isotonic crystalloid to isotonic crystalloid. Trials with a "double intervention," such as those that compared colloid in hypertonic crystalloid to isotonic crystalloid, were analysed separately (for details, see longer version of article on our website). There were no language restrictions.

As the comparison between fluid type was in terms of effects on mortality, we excluded randomised crossover trials. We also excluded trials of preloading in preparation for elective surgery and trials in patients undergoing fluid loading during cardiopulmonary bypass, as in these situations fluids were given for purposes other than volume resuscitation.

Trials were identified by computerised searches of the Cochrane Controlled Trials Register, Medline, Embase, and BIDS Index to Scientific and Technical Proceedings; by hand searching 29 international journals and the proceedings of several international meetings on fluid therapy; by checking the reference lists of trials and review articles; and by contacting the authors of all identified trials, asking them about any other published or unpublished trials that may have been conducted. Further details on the search strategy are available from the authors on request. Eligibility was determined by two reviewers, who also independently extracted the data. Disagreements were resolved by discussion.

\section{Outcome measures and data extraction}

The principal outcome measure was mortality from all causes assessed at the end of the follow up period scheduled for each trial. In trials among surgical patients we sought prospectively gathered information on hospital stay and renal and pulmonary complications as the death rate in these trials was likely to be low (for details, see longer version on our website). For all trials, we extracted information on the type of participants, type of colloid and crystalloid used, duration of follow up, mortality at the end of follow up, and quality of concealment of allocation. We rated quality of concealment of allocation according the criteria proposed by Schulz. ${ }^{10}$ We sought data in simple categorical form, and we did not extract data on time to death. When a report did not include mortality data at all, or when data were incomplete for all patients initially randomised, we sought these data from the trialists.

\section{Data analysis and statistical methods}

Before analysing the results, we identified a number of hypotheses concerning underlying differences in the studies that might require separate analyses or explain heterogeneity in an overall analysis. As efficacy of crystalloids and colloids for resuscitation is thought to differ between different patient types, we stratified the analysis by patients' injury-trauma, burns, surgery, and other conditions, including septicaemia and vascular leak syndrome. Finally, as it has been shown that studies with poor concealment of allocation tend to overestimate the effectiveness of interventions, ${ }^{10}$ we regarded the level of concealment of allocation as a possible source of heterogeneity in study findings. We conducted an additional analysis using only trials with allocation concealment that was known to be adequate.

We calculated relative risks and 95\% confidence intervals for mortality for each trial on an intention to treat basis using the Mantel-Haenszel method. We tested heterogeneity between trials with $\chi^{2}$ tests, with $\mathrm{P} \leqslant 0.05$ indicating significant heterogeneity. When there was no significant heterogeneity we used a fixed effects model to calculate summary relative risks and $95 \%$ confidence intervals for dichotomous data. In the event of significant heterogeneity that could obviously be related to type of injury or allocation concealment, we stratified the analyses on that dimension. As statistical tests of heterogeneity are known to lack power, we also present graphical displays for the summary effect measures of individual trials.

In order to test whether the results of the meta-analyses might have been biased because of selective publication of randomised trials with positive findings (publication bias), we used the regression approach to assessing funnel plot asymmetry proposed by Egger et al. ${ }^{11}$ Using simple unweighted linear regression, the inverse of the variance of each study is regressed against the standard normal deviate. The larger the deviation of the intercept of the regression line from zero, the greater the asymmetry and the more likely it is that the a meta-analysis will yield biased estimates of effect. As suggested by Egger et al, we considered that $\mathrm{P}<0.1$ indicated significant asymmetry.

\section{Results}

We identified a total of 48 apparently randomised trials of fluid resuscitation in critically ill patients, of which 37 met the inclusion criteria. Reasons for exclusion of trials were the use of a crossover design (two trials); testing a resuscitation algorithm (three trials); and the intervention being used for maintaining serum albumin concentrations (three trials), for haemodilution (one trial), for fluid loading (one trial), and for reducing intracranial pressure (one trial).

The table gives key details of the 26 unconfounded trials that compared colloids with crystalloids (see longer version of article on our website for details of the 10 trials that compared colloid in hypertonic crystalloid with isotonic crystalloid and the one that compared colloid in isotonic crystalloid with hypertonic crystalloid). Nineteen of the 26 eligible trials reported mortality. For the other seven trials, we contacted the trialists to ask for any mortality data available for the 307 participants, but no additional information was forthcoming. Our analysis was therefore based on mortality data for 1315 participants from 19 trials. The figure shows that the summary relative risks were similar for all types of injury except surgery, for which the summary measure was imprecise because of small numbers of patients and a low overall mortality (4.7\%).

In four of the trials concealment of allocation was adequate. There was no overall heterogeneity between trials $\left(\chi^{2}=11.67, \mathrm{df}=16, \mathrm{P}=0.75\right)$. The pooled relative risk of death for all patient groups was $1.19(95 \%$ confidence interval 0.98 to 1.45 ). The risk of death in the patients given colloids was $24 \%$ and the risk of death in the patients given crystalloids was $20 \%$, giving an increase in absolute risk of mortality for resuscitation with colloids of $4 \%(0 \%$ to $8 \%)$. The pooled relative risk based only on trials with adequate allocation concealment was 1.29 (0.94 to 1.77), with an increase in absolute risk of mortality for resuscitation with colloids 
Summary of randomised trials comparing colloid and crystalloid fluid resuscitation that met criteria for inclusion

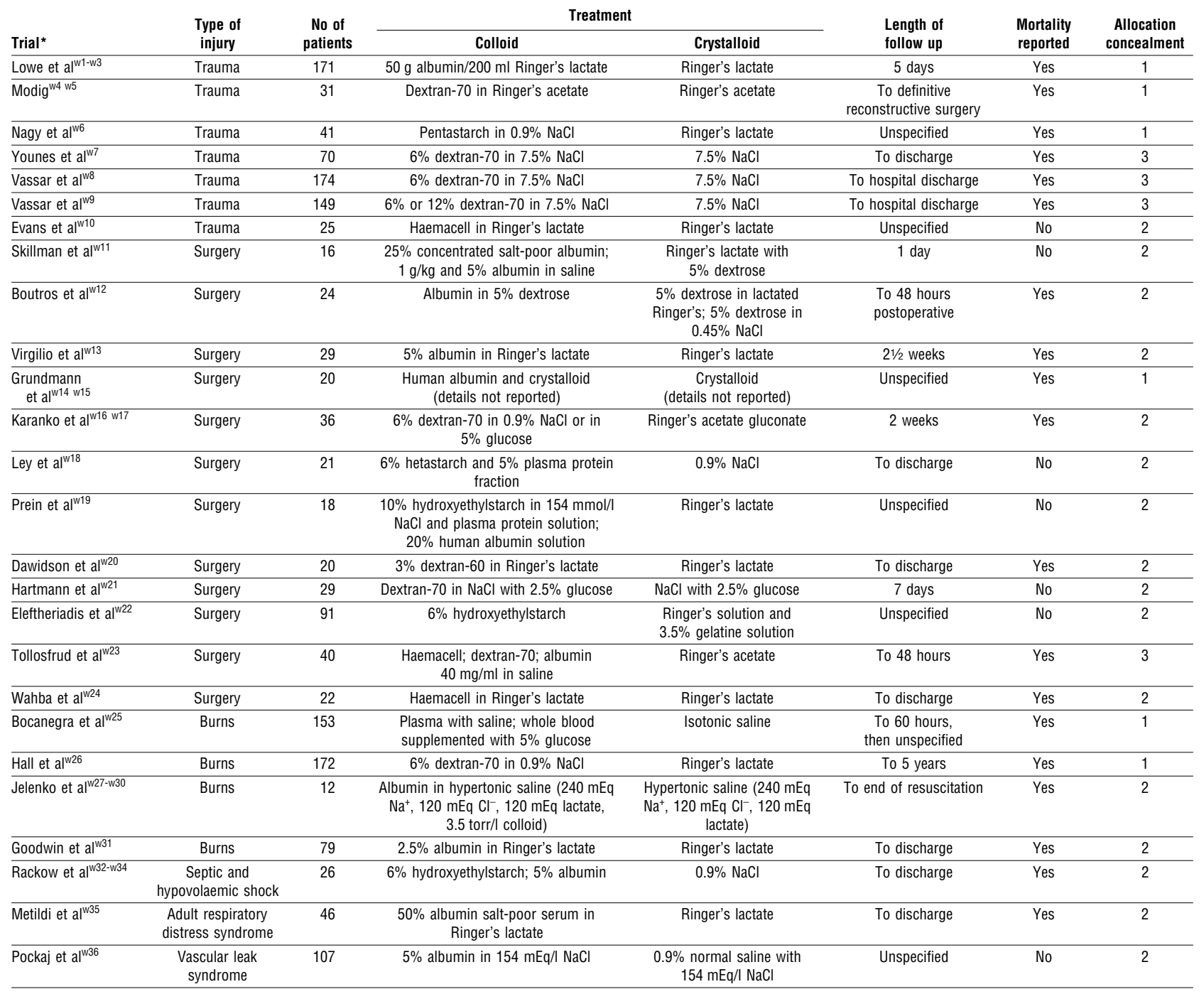

${ }^{\star}$ For list of references see longer version of article on our website www.bmj.com.

of $7 \%(-1 \%$ to $15 \%)$. The regression approach to funnel plot asymmetry yielded an intercept of 0.006 and $\mathrm{P}=0.308$, indicating no statistical evidence for publication bias.

\section{Discussion}

This systematic review synthesised the evidence from randomised controlled trials comparing colloid and crystalloid fluid resuscitation across a wide variety of clinical conditions. There was no statistical evidence of heterogeneity in trial results. The pooled relative risks showed no advantage for resuscitation with colloids, and when we excluded trials with inadequate allocation concealment the pooled relative risk shifted to increased mortality for colloids compared with crystalloids (relative risk 1.29 (95\% confidence interval 0.94 to $1.77))$.

There was no statistical evidence that the effect measure for colloids compared with crystalloids was overestimated because of publication bias. Although the regression test of asymmetry has been shown to have predictive validity, ${ }^{12}$ the few trials in our meta-analysis (19 trials) may mean that detection of such biases would be difficult. Assuming that colloids were the "intervention," publication bias would have resulted in a pooled estimate that understated the extent to which colloids were associated with increased mortality.

\section{Limitations of study}

In common with all meta-analyses, our systematic review may have included studies in which interventions and patient characteristics were sufficiently incomparable that the calculation of a summary effect measure may be questioned. The resuscitation regimen differed between trials, with some trials randomising participants to an initial quantity of colloid or crystalloid and then proceeding with some form of standard resuscitation for all participants, and other trials resuscitating with the randomised fluid to predetermined end points, either resuscitation end points or, in the case of trauma, until corrective surgery. In addition, the type of colloid or crystalloid, the concentration, and the protocol to determine the quantity of fluid varied. 


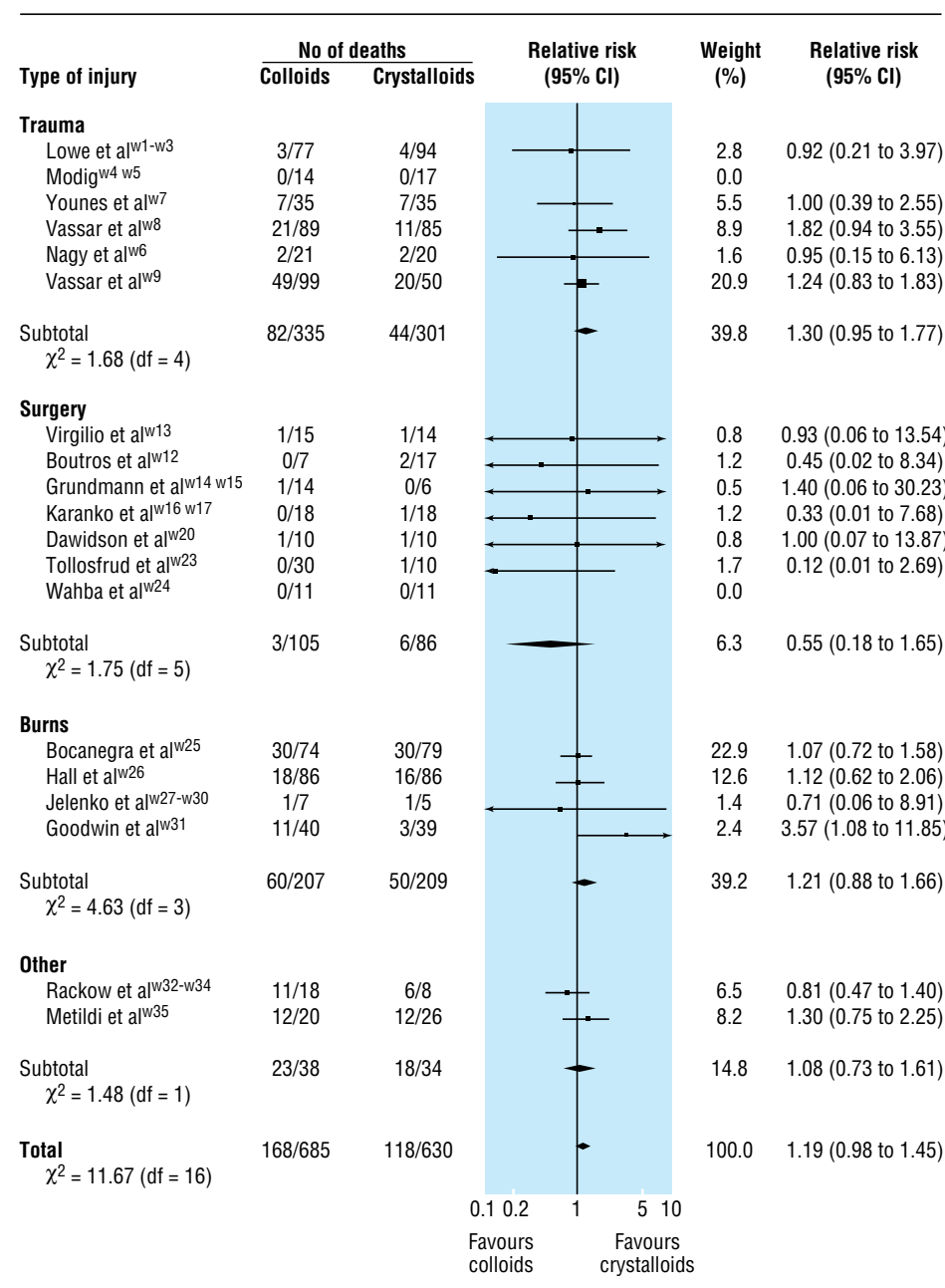

Relative risks (95\% confidence intervals) of death associated with fluid resuscitation with colloid solutions compared with resuscitation with crystalloid solutions

Despite these differences, all participants were in need of volume replacement, and we believe that further "fine tuning" of the intervention would have affected the size of the effect rather than its direction. Although we stratified the analyses by type of injury for which fluid resuscitation was required, these categories are crude and potentially relevant clinical conditions such as uncontrolled haemorrhage or increased capillary permeability might vary widely across and within studies. While this problem could be overcome by analyses of individual patient data from all of the trials, this may not be appropriate as there was little unexplained heterogeneity in the results.

\section{Other studies}

Our results differ from those of Velanovich's metaanalysis of mortality, which concluded that resuscitation with colloids had a beneficial effect on mortality among non-trauma patients compared with crystalloids. ${ }^{7}$ This conclusion was based on three studies of a total of 96 non-trauma patients. Our meta-analysis, based on more than twice the number of patients undergoing surgery (191), failed to support this conclusion. For patients with burns, we also found no evidence for a beneficial effect of colloids. The effect
- For decades there has been controversy over the relative benefits of colloid and crystalloid solutions for fluid resuscitation of hypovolaemic patients

- Although more expensive than crystalloids, use of colloids far exceeds current recommendations

- In this systematic review of randomised controlled trials we found that, compared with crystalloids, use of colloids was associated with an increase in absolute risk of mortality of $4 \%$

- There was no evidence for differences of effect among different types of injury necessitating fluid resuscitation

measure for surgery was extremely imprecise, owing to the small number of patients and a low event rate.

\section{Conclusions}

Resuscitation with colloid solutions was associated with an absolute increase in the risk of mortality of 4\% (95\% confidence interval $0 \%$ to $8 \%$ ), or four extra deaths for every 100 patients resuscitated. As colloids are not associated with improved survival and are considerably more expensive than crystalloids, it is hard to see how their continued use outside randomised controlled trials in subsets of patients of particular concern can be justified.

We thank the Intensive Care National Audit and Research Network in London, for help with identifying trials for this review through their extensive hand searching activities.

Contributors: GS designed the protocol, undertook the literature searches, discussed core ideas about the study design and interpretation of results, and jointly wrote the paper. IR initiated the project, participated in all aspects of the research, and jointly wrote the paper. IR is guarantor for the paper.

Funding: This study was funded by the NHS R\&D Programme: Mother and Child Health.

Conflict of interest: None.

1 Bickell WH, Wall MJ, Pepe PE, Martin R, Ginger VF, Allen MK, et al. Immediate versus delayed resuscitation for hypotensive patients with penetrating torso injuries. N Engl J Med 1994;331:1105-9.

2 Vermeulen LC, Ratko TA, Erstad BL, Brecher ME, Matuszewski KA. A paradigm for consensus. The university hospital consortium guidelines for the use of albumin, nonprotein colloid, and crystalloid solutions. Arch Intern Med 1995;155:373-9.

3 Armstrong RF, Bullen C, Cohen SL, Singer M, Webb AR. Critical care algorithms. Oxford: Oxford Medical Publications, Oxford University Press, 1994.

4 Yim JM, Vermeulen LC, Erstad BL, Matuszewski KA, Burnett DA, Vlasses $\mathrm{PH}$. Albumin and nonprotein colloid solution use in US academic health centers. Arch Intern Med 1995;155:2450-5.

5 Fakhry SM, Alexander J, Smith D, Meyer AA, Peterson HD. Regional and institutional variation in burn care. J Burn Care Rehabil 1995;16:86-90

6 Subcommittee of the Victorian Drug Usage Advisory Committee. Human albumin solutions: an audit of use in three major metropolitan hospitals. Med J Aust 1991;154(10):657-60.

7 Velanovich V. Crystalloid versus colloid fluid resuscitation: a metaanalysis of mortality. Surgery 1989;105:65-71.

8 Bisonni RS, Holtgrave DR, Lawler F, Marley DS. Colloids versus crystalloids in fluid resuscitation: an analysis of randomized controlled trials. $J$ Fam Pract 1991;32:387-90

9 Oxman AD, Cook DJ, Guyatt GH. User's guide to the medical literature. VI. How to use an overview. JAMA 1994;272:1367-71.

10 Schulz KF, Chalmers I, Hayes RJ, Altman DG. Dimensions of methodological quality associated with estimates of treatment effects in controlled trials. JAMA 1995;273:408-12.

11 Egger M, Minder CE, Davey-Smith G. The meta-analytic funnel plot: useful but underdeveloped and underused. $B M J$ (in press).

12 Schneider M, Davey-Smith G, Egger M. Misleading meta-analysis: an examination of meta-analyses and matching large scale randomised controlled trials. J Eval Clin Pract (in press).

(Accepted 3 December 1997) 